\title{
Artemis: A Stratospheric Planet Finder
}

\author{
H.C. Ford ${ }^{1}$, L.D. Petro ${ }^{2}$, C. Burrows ${ }^{2}$, C. Ftaclas 3 , M.C. Roggemann ${ }^{3}$, and J.T. Trauger ${ }^{4}$ \\ 'Johns Hopkins University, Baltimore, MD 21218, USA \\ ${ }^{2}$ Space Telescope Science Institute, 3700 San Martin Dr., Baltimore, MD 21218, USA \\ ${ }^{3}$ Michigan Technological Univ., 1400 Townsend Dr., Houghton, MI 49931-1295, USA \\ ${ }^{4} J P L, M S$ 183-900, 4800 Oak Grove Dr., Pasadena, CA 91109, USA
}

\begin{abstract}
The near-space environment of the stratosphere is far superior to terrestrial sites for optical and infrared observations. New balloon technologies will enable flights and safe recovery of 2-ton payloads at altitudes of 35 $\mathrm{km}$ for 100 days and longer. The combination of long flights and superb observing conditions make it possible to undertake science programs that otherwise could only be done from orbit. We propose to fly an "Ultra-Hubble" Stratospheric Telescope (UHST) equipped with a coronagraphic camera and active optics at $35 \mathrm{~km}$ to search for planets around 200 of the nearest stars. This ULDB mission will establish the frequency of solar-type planetary systems, and provide targets to search for earth-like planets.
\end{abstract}

\section{The Stratosphere: A Superb Observing Site}

At $35 \mathrm{~km}(115,000 \mathrm{ft})$ the stratosphere is cold $\left(\sim-50^{\circ} \mathrm{C}\right)$, very dry $(<0.3 \mu \mathrm{m}$ precipitable water vapor), and rarified ( 5 mbars pressure). Table 1 compares the atmospheric parameters at a good site like Mauna Kea with atmospheric conditions at an altitude of $35 \mathrm{~km}$.

Table 1. Atmospheric Parameters versus Altitude

\begin{tabular}{|c|c|c|c|c|}
\hline $\mathrm{h}(\mathrm{km})$ & $\mathrm{P}(\mathrm{mbars})$ & $\mathrm{T}\left({ }^{\circ} \mathrm{K}\right)$ & $\rho\left(\mathrm{gm} \mathrm{m}^{-3}\right)$ & $\mathrm{H}_{2} \mathrm{O}$ Vapor $\left(\mathrm{gm} \mathrm{m}^{-3}\right)$ \\
\hline 4 & 680 & 253 & 937 & 0.68 \\
\hline 35 & 4.7 & 222 & 7.4 & 0.00011 \\
\hline
\end{tabular}

Astronomers seek out high and dry mountaintops around the world as sites for observatories. If there were a mountaintop with conditions like the stratosphere, astronomers would want to build telescopes there. Fortunately, ULDBs will make the stratosphere accessible for large telescopes (but not astronomers!).

\section{Fried's Parameter at Mountaintop Observatories}

Fried's parameter $r_{0}$ (the characteristic distance across the wavefront for the phase to change by $\sim 4 / 5 \pi$ ) is commonly used as measure of image quality. The 3.8-m Canada-France-Hawaii Telescope (CFHT), which has a good thermal design and is at an excellent site, typically has an $r_{0}$ of $18 \mathrm{~cm}$ at $500 \mathrm{~nm}$. The corresponding fullwidth at half-maximum (FWHM) of the point spread function (PSF) is $0.7^{\prime \prime}$. The field of view $\theta_{0}$ for phase coherence is $\sim 3^{\prime \prime}$, and the timescale $\tau_{0}$ for phase coherence is $\sim 3.6 \mathrm{msec}$. On exceedingly rare occasions $r_{0}$ might be as large as $36 \mathrm{~cm}$, and the corresponding FWHM as small as $0.35^{\prime \prime}$. Because image degradation is caused by atmospheric turbulence that has a continuous spectrum of sizes for phase coherence, the $0.35^{\prime \prime}$ CFHT PSF is very inferior to a $0.35^{\prime \prime}$ PSF produced by a diffraction limited $0.33-\mathrm{m}$ telescope. The small scale, turbulence-induced fluctuations in the index of refraction diffract light to large angles, producing a halo that extends to several arc seconds around the core of the image. The Strehl ratio, which is the ratio of the peak intensity in the observed image to the peak intensity in a perfect (i.e. diffraction limited) image, is $\leq 1 \%$ for the best CFHT optical images. Consequently, atmospheric seeing limits both the resolution and the contrast. 
Intuition suggests that the "atmospheric seeing" should be superb when there is almost no atmosphere above the telescope. This simple idea that "very high must be very good" is bome out by Ftaclas and Roggemann's analysis (2000; hereafter FR00) of data derived from observations that were made from the MIR spacecraft. The observations were measurements of scintillation in stars that were being occulted by the Earth's atmosphere. Grechko et al. (1997) used the scintillation measurements to derive the rms fluctuations in the temperature, $C_{T}^{2}$, between $30 \mathrm{~km}$ and $66 \mathrm{~km}$. FR00 used a model atmosphere (MSIS, 1990; Hedin, 1991) and Eq. 1 to convert $C_{T}^{2}$ to the rms fluctuations in the index of refraction, $C_{n}^{2}$.

$$
C_{n}^{2}=\left(79 \times 10^{-6} \frac{P}{T^{2}}\right)^{2} C_{T}^{2}
$$

The pressure $P$ and temperature $T$ in equation 1 are measured in mbars and degrees Kelvin; the units for $C_{n}^{2}$ are $\mathrm{m}^{-2 / 3}$. Figures 1 and 2 (FR00) show the derived values of $C_{T}^{2}$ and $C_{n}^{2}$ for altitudes between $30 \mathrm{~km}$ and $66 \mathrm{~km}$.

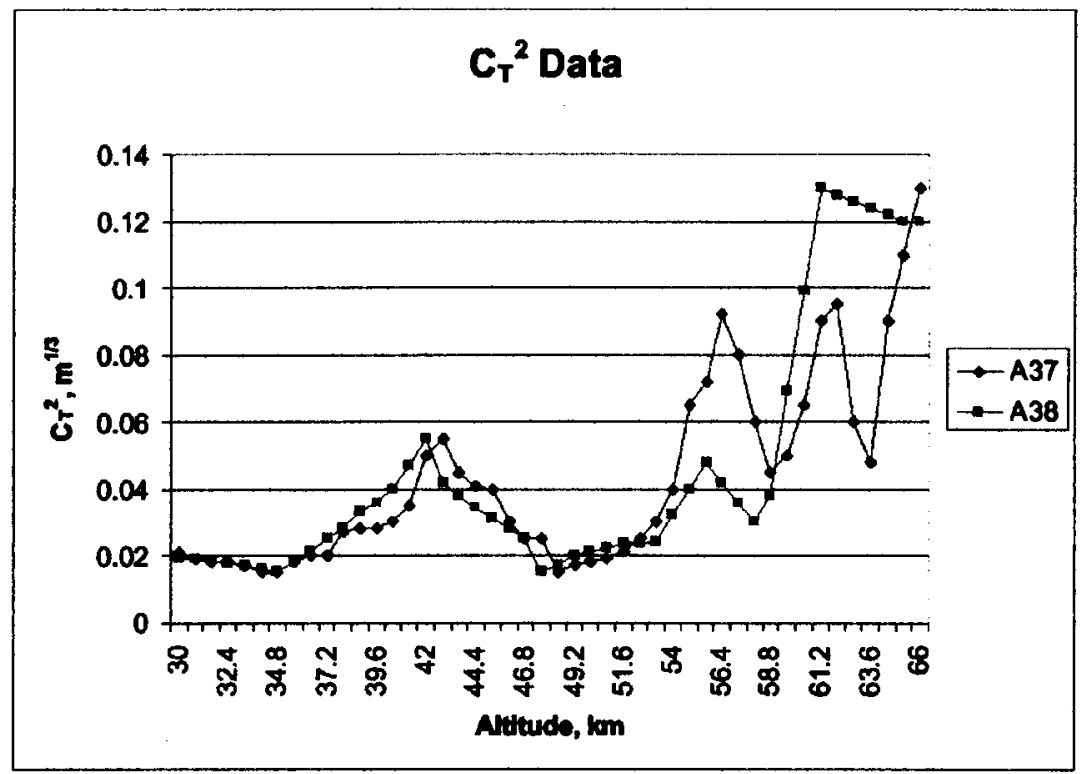

Fig. 1. $C_{\bar{T}}$ versus altitude (trom FKUU) denved trom MIK scintillation observations.

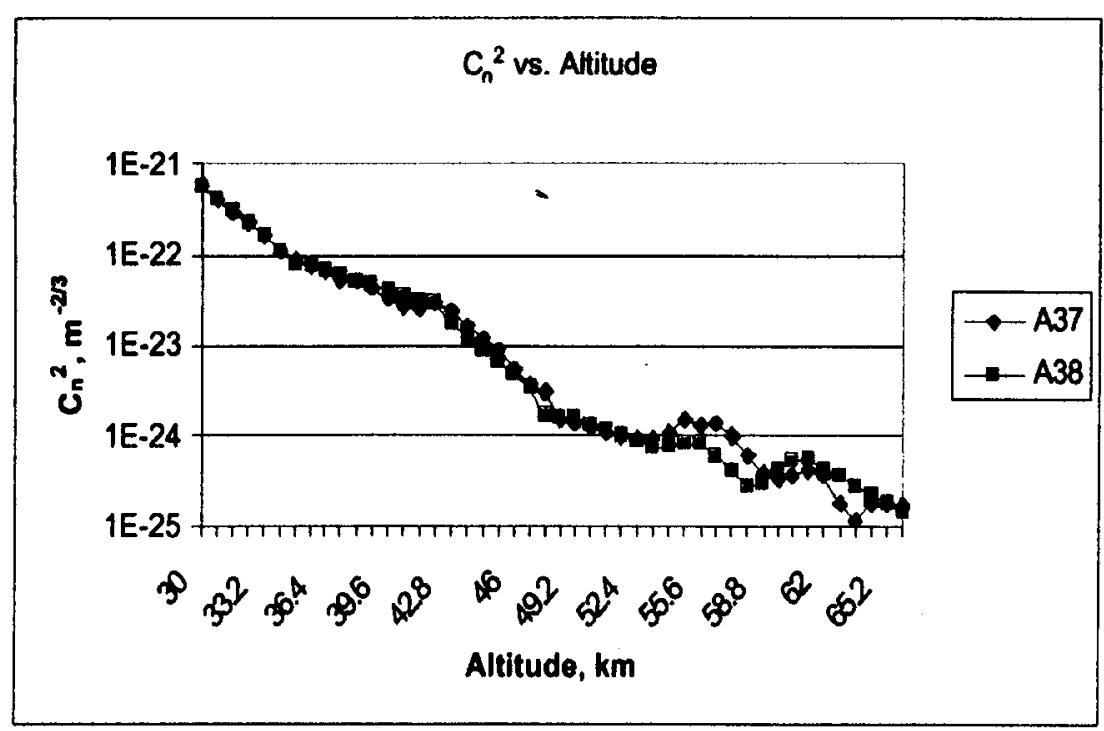

Fig. 2. $C_{n}^{2}$ versus altitude derived by FR00 from the data in Fig. 1.

The labels A37 and A38 refer to the two datasets in Grechko et al. (1997) that extended to altitudes of $66 \mathrm{~km}$. 
When the atmospheric turbulence follows a Kolmogorov spectrum, $r_{0}$ in meters is given by Eq. 2 .

$$
r_{0}=0.185\left[\frac{4 \pi^{2}}{k^{2} \sec \theta_{z} \sum_{m=1}^{M} C_{n m}^{2} \Delta z_{m}}\right]^{3 / 5}
$$

In equation 2 the wave number is $k=2 \pi / \lambda$, the wavelength $\lambda$ is measured in meters, and the summation is over $M$ layers of thickness $\Delta z_{m}$, measured in meters. The structure constant $C_{n}^{2}$ in the $m$-th layer is $C_{n m}^{2}$. The angle between the zenith and the line-of-sight is $\theta_{z}$. FR00's integration of Eq. 2, using the data in Figure 2, gives $r_{0}=250$ meters at $500 \mathrm{~nm}$ for a telescope at an altitude of $30 \mathrm{~km}$ ! Table 2 compares $r_{0}$ and the image quality for telescopes at an altitude of $4 \mathrm{~km}$ (Mauna Kea) and $35 \mathrm{~km}$. Telescopes as large as a 10-m (or larger) will be diffraction limited at ULDB altitudes.

Table 2. A Comparison of Image Quality at $4 \mathrm{~km}$ and $35 \mathrm{~km}$.

\begin{tabular}{|c|c|c|c|c|c|}
\hline Site & Telescope/Aperture & $r_{0}(\mathrm{~m})$ & FWHM (") & $\theta_{0}\left({ }^{\prime \prime}\right)$ & $\tau_{0}(\mathrm{sec})$ \\
\hline Mauna Kea & CFHT 3.8-m & 0.18 & 0.7 & 3 & 0.0036 \\
\hline $35 \mathrm{~km}$ & $2.4-\mathrm{m}$ & $\sim 250$ & 0.048 & $\sim 600$ & $\sim 5$ \\
\hline $35 \mathrm{~km}$ & $10-\mathrm{m}$ & $\sim 250$ & 0.012 & $\sim 600$ & $\sim 5$ \\
\hline
\end{tabular}

\section{Advantages of ULDB Flights Relative to Satellites}

There are several advantages of flying optical telescopes on ULDBs instead of on satellite buses. Because the acceleration in a balloon launch is only a little more than 1-g, the payload does not need to be designed, engineered, and tested to withstand the accelerations, vibrations, and acoustical loads of a launch on a rocket or in the Space Shuttle, although recovery will impose significant accelerations. Consequently, the balloon payload can be much less expensive than an equivalent payload that would be put into orbit. Another advantage is that balloons are much less expensive than launch vehicles. The cost of putting a metric ton into low earth orbit (LEO) is $\sim$ \$50M to $\$ 60 \mathrm{M}$. The cost of a ULDB 100-day mission will be $\sim \$ 3 \mathrm{M}$, which includes the balloon, shipping to Australia, launch, and 100 days of balloon operation (excluding operation of the science payload). Unlike satellites, ULDB balloon payloads can be recovered and reflown. Payload recovery will allow investigators to improve the performance of the gondola and telescope systems by correcting any problems that are found during the first flight. The performance of the telescope and its instruments can be "tweaked or tuned" based on performance the first or second flights. Finally, new technology can be incorporated as it becomes available.

For some missions such as a high resolution, high contrast optical telescope, the stratosphere will be a better site than low earth orbit. The approximately 12-hour long nights will provide a thermal environment that is much less variable than the thermal environment of a satellite that makes a day/night transition every 45 to 50 minutes. Unlike satellites in LEO, ULDB payloads will not be exposed to the damaging radiation of the South Atlantic Anomaly.

\section{Mission Overview: The Payload}

Before committing an expensive high-resolution telescope to a 100-day flight on an ultra long duration balloon flight, we first plan to fly a lightweight, 1-m pathfinder telescope on a 20-day flight that will make one circuit around the earth, returning to or near to the Australian launch site for payload recovery. The goal of the pathfinder is to validate critical telescope and gondola systems. These include: pointing accuracy and pointing stability, wavefront sensing and wavefront control, control of the telescope and balloon's thermal environment, communication and payload control, and power generation and power storage.

The second step in the program will be flight of a 2-m to 3-m "Ultra-Hubble" Stratospheric Telescope equipped with a coronagraphic camera, a wavefront sensor, and active optics for wavefront control. We plan approximately five 100-day UHST flights from Australia. As already noted several flights will allow us to improve the performance of the telescope and gondola system by correcting problems found in the early flights. Stretching 
the flights over several years has other advantages. Multiple flights will allow us to survey all of the nearest stars that are found in the southern sky.

\section{Requirements for the Gondola (i.e. the Telescope Platform)}

Table 3 below lists the most important requirements for the gondola.

Table 3. Gondola Requirements

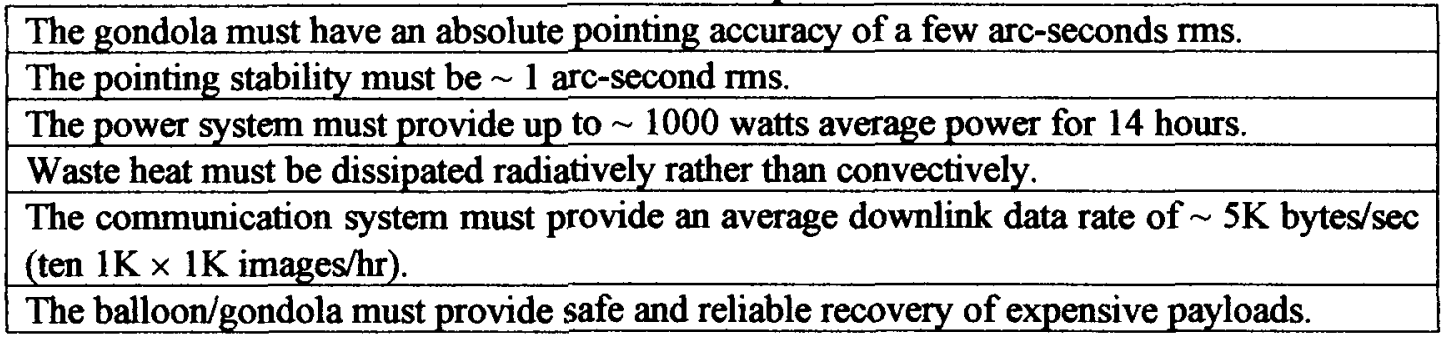

The last requirement in Table 1 is one of the most interesting and important when flying very expensive payloads. High quality-control when the balloon is manufactured will reduce the risk of unexpected flight termination due to a mechanical failure in the hull. As discussed in Knock et al.'s paper in this volume, new technology makes balloon navigation possible for the first time. A lightweight vertical "wing" suspended on a tether can be lowered up to $10 \mathrm{~km}$ below the balloon into winds that have a different velocity than the winds in which the balloon floats. A rudder at the end of the horizontal boom that supports the vertical wing and a counter-balancing "control pod" can fly the wing out to one side (or the other) of the balloon. The airfoil then exerts a force on the balloon, enabling it to be steered. By combining the balloon's known track with predictions of high altitude winds, it will be possible to control the latitude of the balloon. Westward trajectories from Australia at approximately constant latitude will cross Madagascar, southern Africa, and South America at the latitudes of northern Argentina and Chile, and then return to mid-Australia. Such a trajectory minimizes the time the balloon spends flying over densely populated areas, and thus minimizes the probability of dropping the payload into a city. A constant latitude trajectory from Australia will keep the balloon over water $75 \%$ of the time.

Both NASA and the Defense Department have developed Parasails that support heavy loads. Using this technology on balloons should greatly improve the chances of successfully recovering the payload. By combining balloon navigation and parasail descents, a payload could be "flown" to a preselected recovery site such as an airport or a large field. In addition to increasing the chances for a successful payload recovery, a parasail would enable the payload to be flown away from a populated area in the event of a catastrophic failure of the balloon.

Because ULDBs will spend $75 \%$ of their flight time over water, it is highly desirable to develop a lightweight, watertight shell that can enclose and seal the payload if a landing in the water should happen.

\section{Telescope/Coronagraph Requirements}

The high level requirements for the telescope and coronagraph are summarized in Table 4.

Table 4. Requirements for the UHST and Coronagraph

The telescope must be an off-axis paraboloid with a diameter between 2 and 3 meters.

The telescope must have an image rotator.

The telescope must provide an internal tip-tilt stabilization of $\sim 0.003^{\prime \prime} \mathrm{rms}$.

An apodized coronagraph and deformable mirror must provide sufficient contrast to detect Jupiter-like planets at star-planet separations $\mathrm{R}_{\text {star-planet }} \geq 0.5^{\prime \prime}$.

A nulling interferometer must provide sufficient contrast to detect known planets with $R_{\text {star-planet }} \leq 0.1^{\prime \prime}$.

Detection of either Jupiter-like or Earth-like planets in the glare of the parent star puts stringent requirements on the image quality and image contrast provided by the telescope and coronagraph system. A Jupiter at distance of $\sim 5$ astronomical units (AUs), or an earth at $1 \mathrm{AU}$ from its parent star will be $\sim 10^{-8}$ times fainter than the parent star. Consequently, to detect the planet the surface brightness of diffracted and scattered light from the 
parent star within an area comparable to the FWHM of the PSF must be $\leq 10^{-8}$ of the star's total light. This requirement drives most of the requirements for the telescope, coronagraph, and wavefront sensing and correcting system. Because the Airy profile scales as $\lambda / D$, relative short wavelengths and large apertures are desirable. Flying an off-axis paraboloid eliminates diffraction in the pupil plane from the secondary supports and the secondary mirror. Low amplitude, spatially coherent polishing errors on the primary or secondary mirrors, such as those in the primary mirror of the Hubble Space Telescope (Burrows, 1990), diffract light into the near field of the PSF (Brown and Burrows, 1990). Reducing the contrast by a factor of $10^{-8}$ requires that the rms errors in wavefront be corrected to $\sim \lambda / 1000$. Either the primary mirror has to be polished to better than $\lambda / 1000$, or the wavefront sensor and wavefront correction system must be capable of correcting the atmosphere plus telescope wavefront errors to this accuracy.

Whatever the level of the wavefront correct, there always will be a residual diffraction pattern from frequencies that are too high to be corrected by the deformable mirror. The validity of a faint candidate planet will be determined by using an image rotator to rotate the image with respect to the detector. If the candidate moves with respect to the fixed diffraction residuals, the candidate is likely a real source, though not necessarily a planet. If the candidate is detected at a second epoch, and has very nearly the same (large) proper motion as the bright star, it will be a strong candidate for a planet.

Figure 3 shows a simulation of the contrast achievable with present generation deformable mirror and a 2.4$\mathrm{m}$ mirror that has the same polishing errors as the Hubble primary mirror. A telescope performing like the one in Figure 3 would be able to detect the Jupiter-mass planet recently discovered orbiting the nearby star Epsilon Eridani.

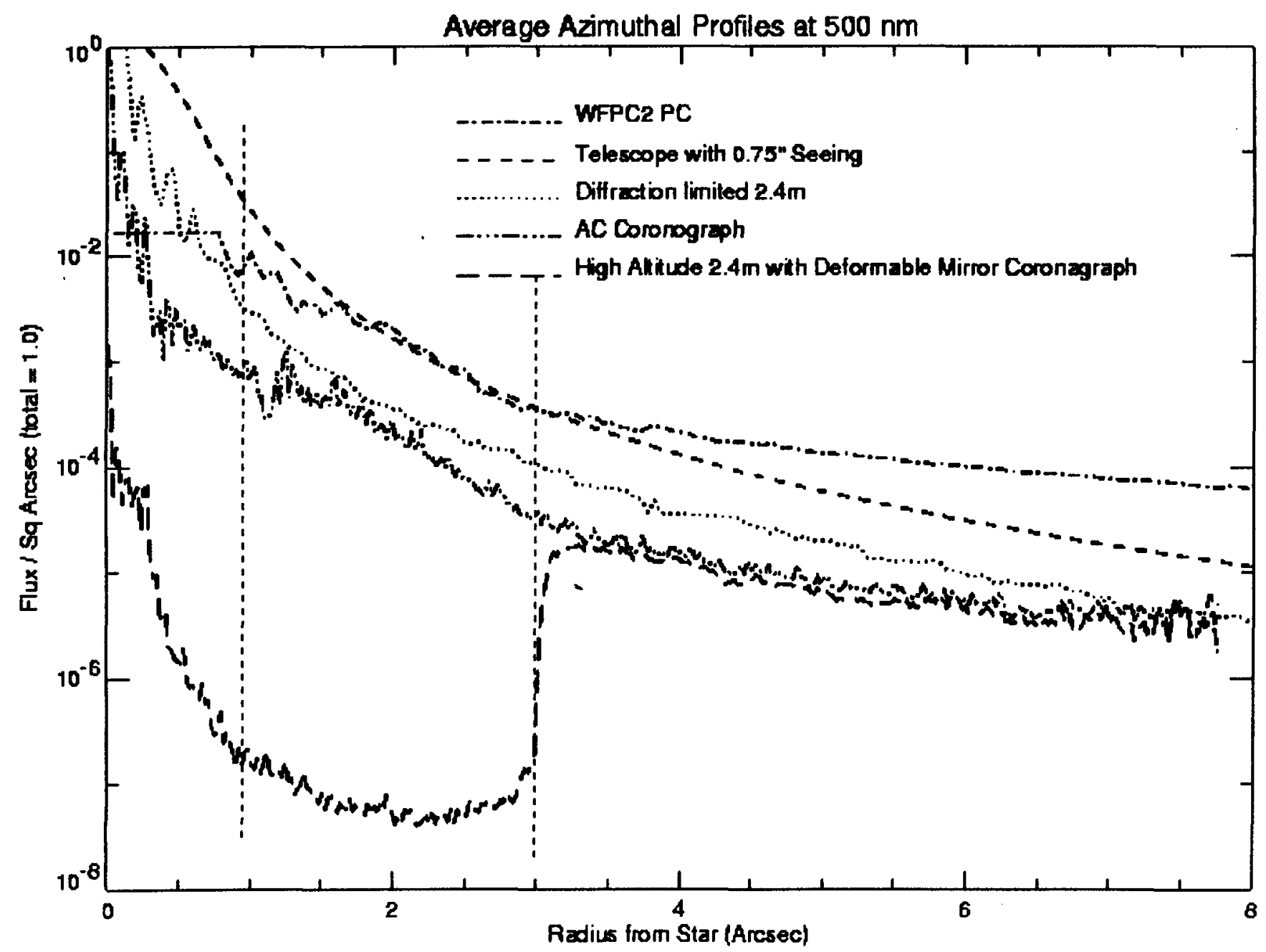

mirror like the one in the Hubble Space Telescope. The vertical dashed line on the right is the limit set by the density of actuators on the deformable mirror. The vertical dashed line on the left is the limit set by the radius of the coronagraphic field stop that was used in the simulations. 


\section{Searching Nearby Stars for Planets}

High precision spectroscopic searches for planets have produced spectacular results (e.g. Marcy and Butler, 1998). At the time of this writing, there are 50 detections of extra-solar planets. Because of the technical difficulty of detecting the small reflex motion of a parent star around the star-planet barycenter, present searches are highly biased toward the discovery of massive planets $\left(0.5 \leq m \sin I \leq 7 \mathrm{M}_{\text {upp }}\right)$ close to the parent star $\left(R_{\text {star-planet }} \leq 2.1 \mathrm{AU}\right)$ . Unexpectedly, planets more massive than Jupiter have been discussed closer to their parent star than is Mercury to the Sun. Spectroscopic techniques are presently incapable of detecting earth-like planets at a star-planet separation of $1 \mathrm{AU}$, or Jupiter or Saturn-like planets at distances between 5 and $10 \mathrm{AU}$.

We propose to fly a 2-m to 3-m UHST on five 100-day ULDB flights from Australia. Our first goal is to observe the $\sim 200$ best candidate stars within $25 \mathrm{pc}$ of the Sun that are visible from the southern hemisphere, searching for planets that are similar to Jupiter and Saturn. We will design the entire system to be able to detect the technically-easiest planets that already have been discovered by high precision radial velocity measurements. We will observe each star at 2 epochs in order to determine whether or not faint candidates share the proper motion of the parent star.

Our long range goal is to use the experience gained from Artemis to design and fly a telescope (on a ULDB) that can both detect earth-like planets around nearby stars, and take spectra of the planets to look for the spectral signature of oxygen. Finding oxygen would be a certain indicator that life is present on the planet. We would concentrate our search for earths on any systems that have Jupiter or Saturn like planets at 5 to 10 AUs from the parent stars. That is, we would take a Galilean view, and assume that a star-planet system that is like our solar system in its outer parts is more likely to have an earth in its inner parts than the systems presently being discovered, which are very different than our solar system.

We conclude by noting that science always advances fastest when many different scientists attack a problem with different techniques and points of view. NASA has in place an expensive, long-range program aimed at finding earth-like planets. We think that a very modest investment in alternative approaches, such as UHSTs on ULDB flights, will bring results that could well help shape the direction of the more expensive, long range programs.

\section{ACKNOWLEDGMENTS}

This research was supported with funds from the GSFC. We are grateful to John Krist for providing Figure 3 from his simulations of a telescope and coronagraph with a Hubble-like mirror and a realistic deformable mirror.

\section{REFERENCES}

Brown, R. and Burrows, C., On the Feasibility of Detecting Extrasolar Planets by Reflected Starlight Using the Hubble Space Telescope, Icarus, 87, 484, 1991.

Burrows, C., Hubble Space Telescope Optical Telescope Assembly Handbook, Version 1, 1990.

Ftaclas, C. and Roggemann, M C., preprint, 2000 (FR00).

Grechko, G. M., Gurvich, A. S., Kan, V., Pakhomov, A. I., Podvyazny, Y. P., and Savchenko, S. A., Observation of Atmospheric Turbulence at Altitudes of $20-70 \mathrm{~km}$, Doclady of Russian Academy of Science, Geophysics, 357, 683-686, 1997 (in Russian).

Hedin, A. E., Extension of the MSIS Thermospheric Model into the Middle and Lower Atmosphere, J. Geophys. Res., 96, 1159, 1991.

Marcy, G. W. and Butler, R. P., Detection of Extrasolar Planets, Ann. Rev. Astron. Astrophys., 36, 57-97, 1998.

MSIS, A NASA Web Site Containing the MSIS-90 Model, http://bolero.gsfc.nasa.gov/cgi-bin/natasha/msis m. 1990. 